\title{
Adipogenic RNAs are transferred in osteoblasts via bone marrow adipocytes-derived extracellular vesicles (EVs)
}

Perrine J Martin ${ }^{1,3,4,5^{*}}$, Nathalie Haren ${ }^{1,2,3}$, Olfa Ghali ${ }^{1,2,3}$, Aline Clabaut ${ }^{1,2,3}$, Christophe Chauveau ${ }^{1,2,3}$, Pierre Hardouin ${ }^{1,2,3}$ and Odile Broux ${ }^{1,2,3}$

\begin{abstract}
Background: In osteoporosis, bone loss is accompanied by increased marrow adiposity. Given their proximity in the bone marrow and their shared origin, a dialogue between adipocytes and osteoblasts could be a factor in the competition between human Mesenchymal Stem Cells (hMSC) differentiation routes, leading to adipocyte differentiation at the expense of osteoblast differentiation. The adipocyte/osteoblast balance is highly regulated at the level of gene transcription. In our work, we focused on PPARgamma, CEBPalpha and CEBPdelta, as these transcription factors are seen as master regulators of adipogenesis and expressed precociously, and on leptin and adiponectin, considered as adipocyte marker genes. In 2010, our group has demonstrated, thanks to a coculture model, that in the presence of hMSC-derived adipocytes (hMSC-Adi), hMSC-derived osteoblasts (hMSC-Ost) express lesser amounts of osteogenic markers but exhibit the expression of typical adipogenic genes. Nevertheless, the mechanisms underlying this modulation of gene expression are not clarified. Recently, adipocytes were described as releasing extracellular vesicles (EVs), containing and transferring adipocyte specific transcripts, like PPARgamma, leptin and adiponectin. Here, we investigated whether EVs could be the way in which adipocytes transfer adipogenic RNAs in our coculture model.

Results: We observed in hMSC-Ost incubated in hAdi-CM an increase in the adipogenic PPARy, leptin, CEBPa and CEBP $\delta$ transcripts as well as the anti-osteoblastic miR-138, miR30c, miR125a, miR-125b, miR-31 miRNAs, probably implicated in the observed osteocalcin (OC) and osteopontin (OP) expression decrease. Moreover, EVs were isolated from conditioned media collected from cultures of hMSC at different stages of adipocyte differentiation and these specific adipogenic transcripts were detected inside. Finally, thanks to interspecies conditioned media exposition, we could highlight for the first time a horizontal transfer of adipogenic transcripts from medullary adipocytes to osteoblasts.

Conclusions: Here, we have shown, for the first time, RNA transfer between hMSC-derived adipocytes and osteoblasts through EVs. Additional studies are needed to clarify if this mechanism has a role in the adipocytic switch driven on osteoblasts by adipocytes inside bone marrow and if EVs could be a target component to regulate the competition between osteoblasts and adipocytes in the prevention or in the therapy of osteoporosis and other osteopenia.
\end{abstract}

Keywords: Extracellular vesicles, RNA transfer, Adipocytes, Osteoblasts, Osteoporosis

\footnotetext{
* Correspondence: perrine.martin@univ-littoral.fr

'Univ Lille Nord de France, F-59000 Lille, France

${ }^{3}$ Université du Littoral Côte d'Opale, ULCO, F-62327 Boulogne sur Mer,

France

Full list of author information is available at the end of the article
}

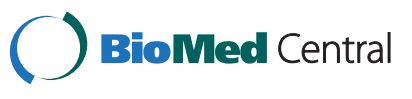

(c) 2015 Martin et al.; licensee BioMed Central. This is an Open Access article distributed under the terms of the Creative Commons Attribution License (http://creativecommons.org/licenses/by/4.0), which permits unrestricted use, distribution, and reproduction in any medium, provided the original work is properly credited. The Creative Commons Public Domain Dedication waiver (http://creativecommons.org/publicdomain/zero/1.0/) applies to the data made available in this article unless otherwise stated. 


\section{Background}

Maintenance of healthy bone mass requires a continuous process of bone renewal, known as bone remodelling, which consists of a balance of bone resorption by osteoclasts and bone formation by osteoblasts. The decrease in bone mass that occurs in osteoporosis results from the impairment of this balance, leading to increased fracture risk. Additional evidence from in vitro, in vivo and clinical studies also supports a link between bone loss and accumulation of medullary adipocytes [1-5]. Situations such as aging, estrogen insufficiency [6], anorexia nervosa [7], diverse therapies [8], microgravity exposure [9], or factors such as miRNAs [10,11] are known to induce bone loss concurrently with enhanced bone marrow adiposity. Evidence shows that a dialogue between adipocytes and osteoblasts is one of the mechanisms occurring in the competition between human Mesenchymal Stem Cells (hMSC) differentiation routes, supporting adipocyte differentiation at the expense of osteoblast differentiation. Consequently, factors which promote adipogenesis not only lead to "fatty marrow" but also inhibit osteoblastogenesis or osteoblast proliferation, resulting in decreased osteoblast numbers, diminished bone formation and, potentially, loss of bone mass leading to osteoporosis $[12,13]$. The adipocyte/osteoblast balance is highly regulated at the level of gene transcription [14]. It is difficult to find specific adipogenic mRNAs that are not shared by osteoblasts $[15,16]$. In our work, we focused on one side on PPAR $\gamma$, CEBP $\alpha$ and CEBP $\delta$, as these transcription factors are seen as the master regulators of adipogenesis [17] and are expressed precociously, and on the other side on leptin and adiponectin, considered as adipocyte marker genes increasing in a time-dependent manner during adipogenic induction [15].

In 2010, our group has demonstrated, using a coculture model [18], that in the presence of hMSC-derived adipocytes (hMSC-Adi), hMSC-derived osteoblasts (hMSC-Ost) express lower amounts of osteogenic markers but exhibit expression of typical adipogenic genes. Nevertheless, the mechanisms underlying this modulation of gene expression are not clarified. Extracellular Vesicles (EVs) are $100 \mathrm{~nm}$ to $1 \mu \mathrm{m}$ membrane-bound structures released from the plasma membrane of most cell types and are involved in a range of physiological processes, including angiogenesis, inflammation, progression of cancers and reprogramming of mesenchymal stem cells, especially by transferring RNAs [19]. Recently, primary rat and cultured mouse adipocytes were described as releasing EVs [20]. They have been shown to contain adipocyte specific transcripts, like leptin and adiponectin, that are both transferred into and expressed in acceptor adipocytes and are involved in the upregulation of lipogenesis and cell size [21]. Moreover, adipocyte-derived vesicles were demonstrated to transfer adipocyte-specific gene transcripts such as adiponectin, resistin, and PPAR 22 into RAW264.7 macrophages [22].

Here, we considered if EVs could be the mechanism by which adipocytes transfer adipogenic RNAs in our coculture model. To confirm this hypothesis, we incubated hMSC-Ost in conditioned medium obtained from hMSC-Adi (hAdi-CM) cultures. We observed in the osteoblastic population an increase in the adipogenic PPAR $\gamma$, leptin, CEBP $\alpha$ and CEBP $\delta$ transcripts, dependent on mRNA amount as shown by conditioned media obtained from adipocytes at several differentiation stages and PPAR $\gamma$ silencing experiments, as well as the antiosteoblastic miR-138, miR30c, miR125a, miR-125b, miR-31 miRNAs [23-26], probably implicated in osteocalcin (OC) and osteopontin (OP) expression decrease. Moreover, we isolated EVs from conditioned media collected from cultures of hMSC at different stages of adipocyte differentiation and detected inside the presence of these specific adipogenic transcripts. Finally, thanks to inter-species conditioned media exposition, and using species specific primers, we could highlight for the first time a horizontal transfer of adipogenic transcripts from medullary adipocytes to osteoblasts. Here, we postulate that hMSCderived adipocytes are able to produce EVs responsible for the transfer of adipogenic RNAs to osteoblasts.

\section{Results}

Adipocyte-specific RNA expression is increased in osteoblasts by hMSC-derived adipocyte conditioned media and influence osteoblast markers expression hMSC-Ost and hMSC-Adi were obtained as described in Materials and Methods. We examined if hMSC-Ost incubation during $48 \mathrm{~h}$ in conditioned media issued from hMSC-Adi (hAdi-CM) after 0, 7, 14 and 21 days of differentiation, lead to a variation of adipocyte and osteoblast specific gene expression in osteoblasts. First, we measured by RT-qPCR expression levels of the four adipocytespecific genes PPAR $\gamma$, leptin, CEBP $\alpha$ and CEBP $\delta$ and relative quantification was performed on the reference gene YWHAZ. As shown in Figure 1A, relative expression levels of the four genes were directly dependent on the stage of differentiation of the hMSC-Adi. The maximal increase was reached with the CM obtained after 14 days of adipocyte differentiation for all of the genes. For this reason, miRNA and osteoblasts mRNA levels were measured in hMSC-Ost incubated in conditioned medium from adipocytes differentiated for 14 days. Five miRNAs, miR-138, miR-30c, miR-125a, miR-125b and miR-31, were selected for their capacity to inhibit osteoblast gene expression [25-28]. As shown in Figure 1B, all of them showed an increase in their expression in hMSC-Ost incubated with 14 days differentiated hAdi-CM. Furthermore, incubation of hOst D14 with hAdi D14 CM led to a slight but 


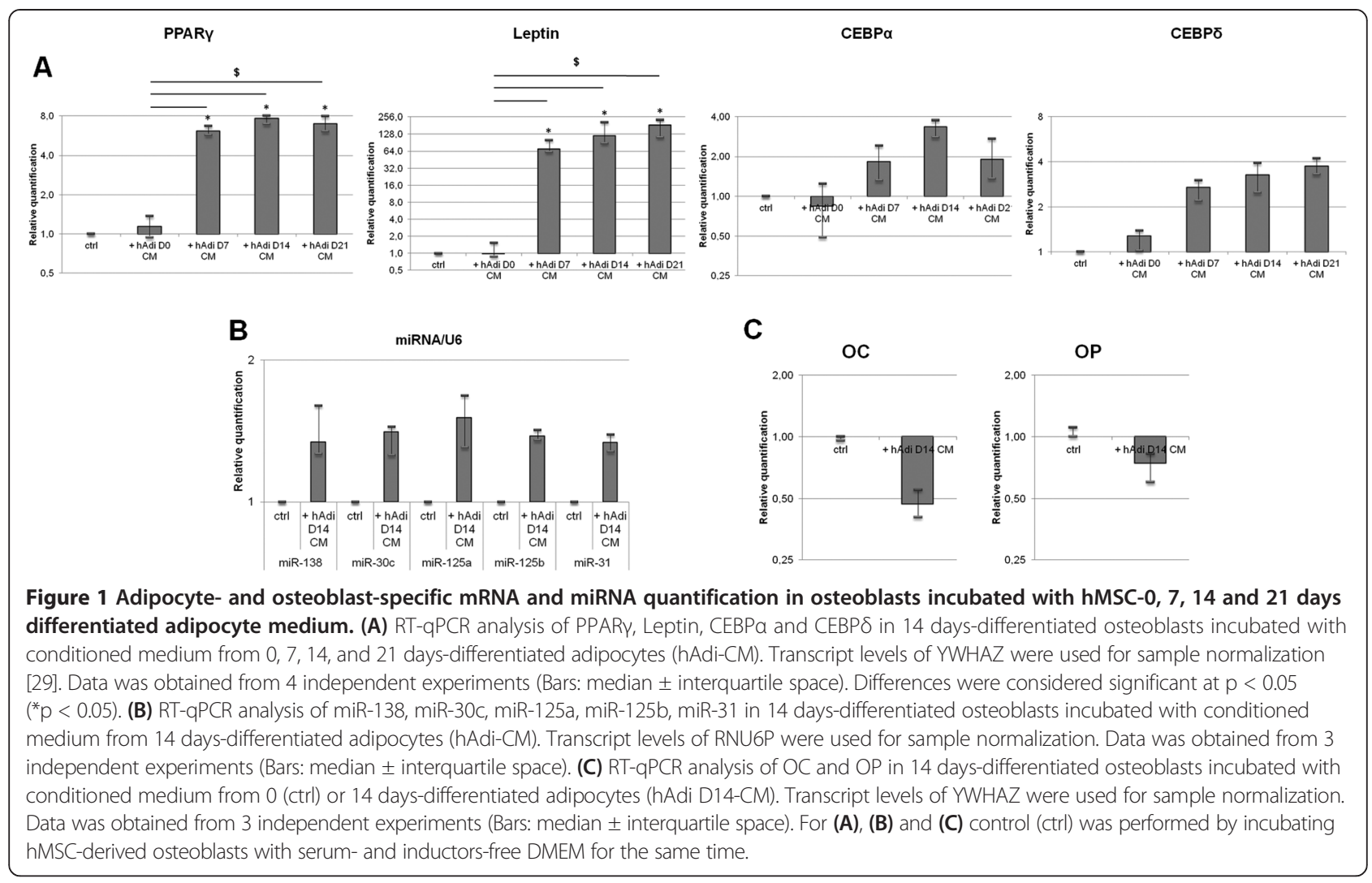

reproducible decrease of two late osteoblastic differentiation markers of gene expression, osteocalcin (OC) and osteopontin (OP) (Figure 1C), supporting the idea that at least some of the transferred miRNAs are functional.

Taken together, these results indicate that some compounds are secreted by differentiated hMSC-Adi leading to an increase in specific pro-adipogenic or antiosteoblastic RNAs in osteoblasts.

\section{hMSC-Adi express adipogenic mRNAs and anti-osteoblastic miRNAs proportionally as those found in hMSC-Ost incubated in hAdi-CM}

We wanted to confirm the increase in the same specific RNAs in hMSC-derived adipocytes at 0, 7, 14 and 21 days of differentiation as in osteoblasts incubated with the corresponding conditioned media. Figure 2A shows representative images of the differentiating hAdi used for the generation of CM. Appearance of lipid droplets after 7 days of differentiation whose number increases at 14 and 21 days were observed, as expected. In addition, Figure $2 \mathrm{~B}$ shows that hMSC-Adi expressed increasing amounts of the four studied adipogenic mRNAs, depending on the stage of differentiation. More precisely, PPAR $\gamma, \mathrm{CEBP} \alpha$ and CEBP $\delta$ reached a maximal level of expression after 7 days of differentiation whereas the maximal level of leptin expression was reached after
14 days of differentiation, with no mRNA detected at day 0 . Concerning the studied miRNAs, their expression was enhanced in 14 days differentiated hMSC-Adi (Figure 2C).

Furthermore, silencing experiments were performed to examine if knockdown of PPAR $\gamma$ mRNA in the donor adipocytes leads to a reduction of the corresponding RNA in the recipient osteoblasts after conditioned medium transfer. hAdi D14 were transfected with siRNA targeting PPAR $\gamma$ mRNA and a substantial decrease (more than 95\%) could be obtained (Figure 2D). Incubation of hOst D14 with the subsequent conditioned medium (hAdi D14 siPPAR $\mathrm{CM}$ ) really led to a reduction of PPAR $\gamma$ mRNA in the recipient osteoblasts compared to those incubated with hAdi D14 CM (Figure 2E).

In conclusion, the expression of RNAs found in hMSC-Ost incubated with hAdi-CM follows the same tendency as those found in hMSC-Adi from which the $\mathrm{CM}$ is obtained, suggesting RNA transfer between the two cell types.

\section{Extracellular vesicles harbouring adipogenic mRNAs are found in hAdi-CM}

We wanted to investigate if EVs can be one of the vectors taking part in the adipocyte gene rise described above. As a first confirmation, hMSC-Adi EVs were 


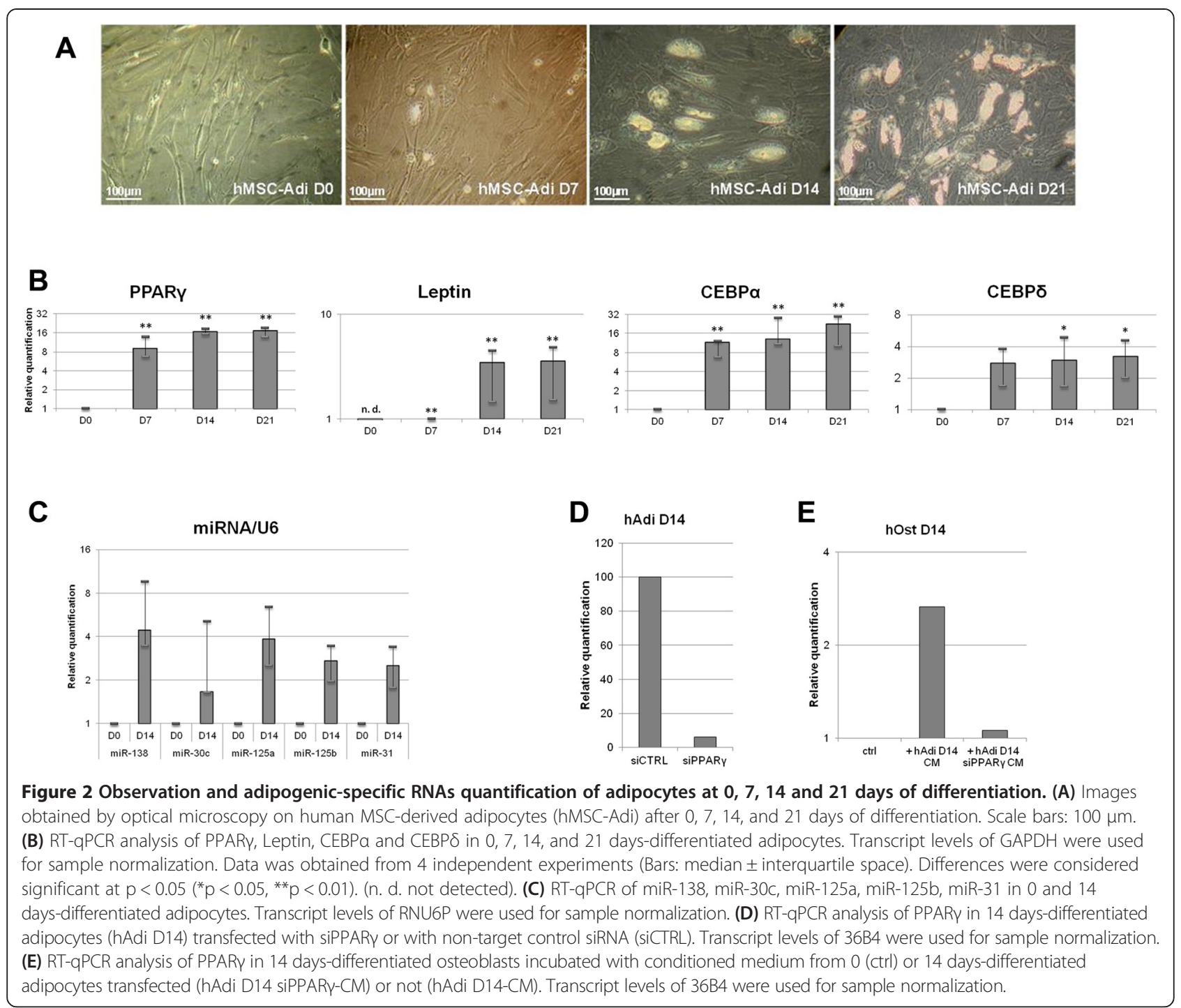

successfully isolated and purified from $\mathrm{CM}$ using the method of differential centrifugation and filtration [30]. The obtained hMSC-Adi EVs were observed under transmission electron microscopy, which revealed that they are approximately $30-100 \mathrm{~nm}$ in size (Figure 3A). RNAs were then isolated from these EVs at the differentiation times of their adipocytes of origin. As shown in Figure 3B, RT-qPCR experiments revealed that EVs hold as proportional amounts of specific mRNA as hMSCAdi expressed depending on the differentiation stage. For example, no mRNA coding for leptin was detected in EVs isolated from the non-differentiated adipocyte (day 0) CM, as found in the cells of origin (Figure 2B, day 0 ). We also noticed the same maximum increase at day 7 of differentiation, maintained at day 14 and 21, of CEBP $\alpha$ and CEBP $\delta$ mRNAs in EVs as in hMSC-Adi. Finally, we observed a similar expression of each of these mRNAs in EVs and in hMSC-Ost incubated with hAdi-CM. These results suggest that EVs could be the vehicle by which adipogenic mRNAs are transferred to osteoblasts.

\section{Species-specific adipogenic mRNAs are transferred from adipocytes to osteoblasts}

To carry out inter-species experiments, hMSC-Adi CM was applied to the mouse cell line MC3T3-derived osteoblasts and RT-qPCR was performed with highly specific human primers against adipocyte specific genes. Each of these primers were tested on mouse RNA for their specificity (data not shown). hGAPDH and h36B4 genes, usually used as reference genes, were chosen as they are known to be commonly found in EVs [19]. As shown in Figure 4A, the human adipocyte genes hPPAR, hLeptin and hCEBP $\delta$ were specifically amplified from RNA 


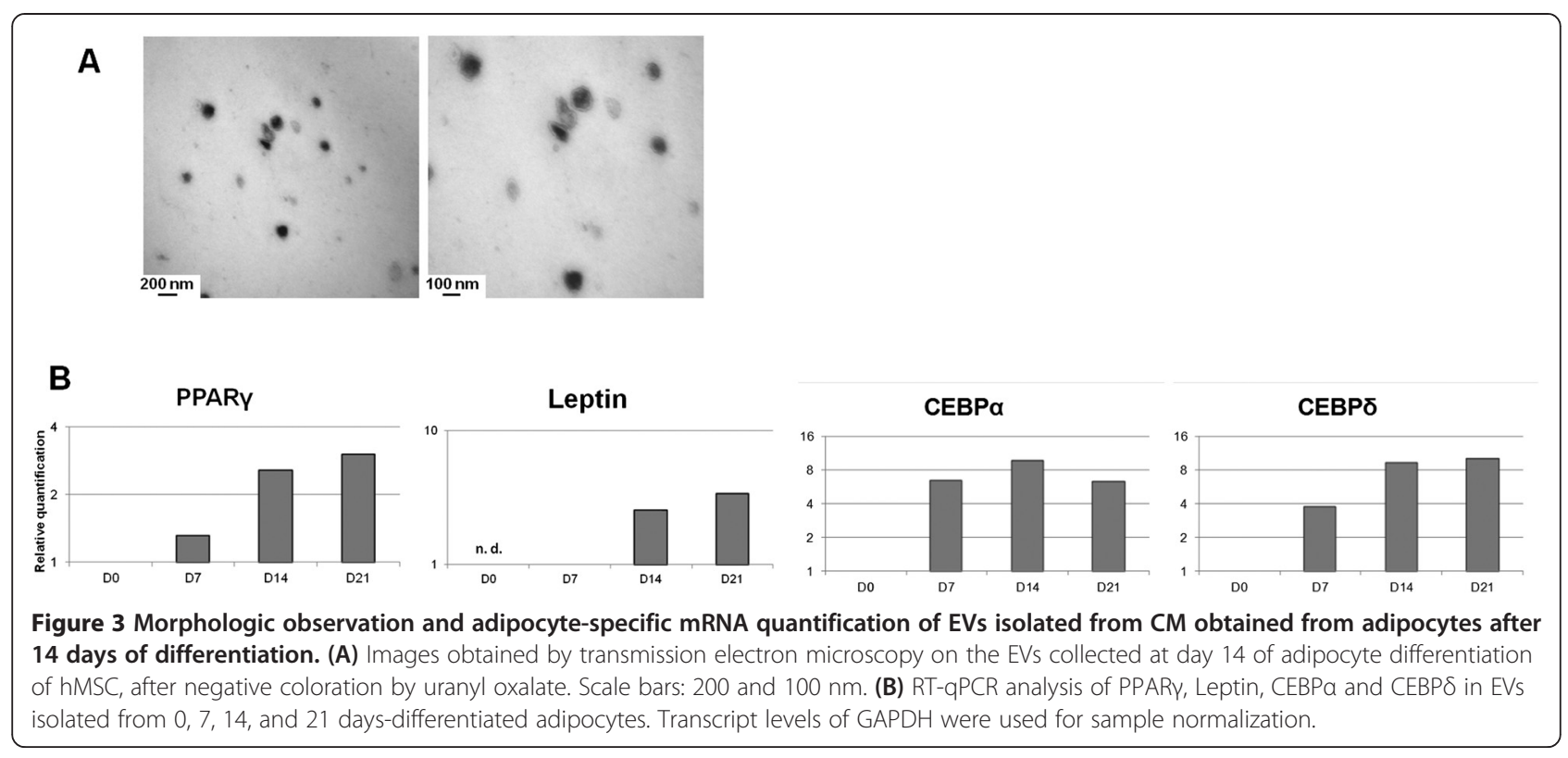

isolated from MC3T3. Similarly, hGAPDH and h36B4. RNAs were found to be amplified. These results confirm the hypothesis of a mRNA transfer from adipocytes to osteoblasts.

To strengthen these results, the reverse experiment was performed. Mouse bone marrow primary cells were obtained as described in Materials and Methods and were differentiated into adipocytes during 14 days (mBM-Adi D14). Observation by optical microscopy (Figure 4B) showed the appearance of lipid droplets confirming the adipocytic differentiation. Conditioned medium of mBMAdi D14 (mBM-Adi D14 CM) was applied to hMSC-Ost and amplification of the mouse genes mPPAR $\gamma$, mAdiponectin and mCEBP $\alpha$ was performed using highly specific primers as well (Figure 4C). Although a non-specific amplicon, especially for mPPAR $\gamma$ and mAdiponectin, appeared for hMSC-Ost D14, this happened in all conditions, confirming the non-specificity of the band. For both mRNA, a specific band at the right size is observed, only when hMSC-Ost D14 were incubated with mBM-Adi D14 CM. Moreover mBM-Adi D14 were used as a positive control for mouse genes amplification, showing a unique band at the corresponding size, confirming the specificity of the primers for the mouse PPAR $\gamma$ and Adiponectin mRNAs. mGAPDH and mActin genes were used as reference genes commonly found in EVs [19]. Taken together, these results highlighted that the three adipocyte specific mouse mRNAs mPPAR $\gamma$, mAdiponectin and mCEBP $\alpha$ as well as mGAPDH and mActin were found in hMSC-Ost incubated with mBM-Adi D14 CM, supporting transfer of mRNAs from adipocyte derived from bone marrow primary cells.

\section{Discussion}

The increase in marrow adipocytes observed in osteoporosis may contribute to limiting of osteoblast commitment by acting on MSCs or even directly on osteoblasts [31]. This hypothesis is supported by the proximity of adipocytes to osteoblasts in the bone marrow, their shared mesenchymal progenitor origin, and the fact that adipocytes are secretory cells. In an attempt to reproduce cellular interactions within the bone marrow, our group previously developed a coculture system using hMSC-derived osteoblasts and adipocytes [18]. In this model, a negative influence of adipocytes on osteoblast differentiation has been observed, supporting this assumption. Bone marrow adipocytes are highly secreting cells and, amongst factors such as fatty acids and adipokines already described in vitro to modify their neighbouring cells [32-34], we wonder if other mediators could be implicated. Within the past decade, EVs have emerged as important factors in intercellular communication, being involved in the transmission of biological signals between cells $[19,35]$. EVs components may include proteins, mRNAs and miRNAs. These components can be delivered to neighbouring cells and can be functional in their new location. The protein and RNA composition of EVs depends on the cell type or tissue source from which they originate. Although EVs are released from many cell types, to our knowledge no study has reported their secretion by hMSC-derived adipocytes.

In this study we could show that hMSC-Adi secrete EVs containing adipocyte specific mRNAs and antiosteogenic miRNAs, that can be transferred to osteoblasts. Morphologically, the obtained EVs look quite 


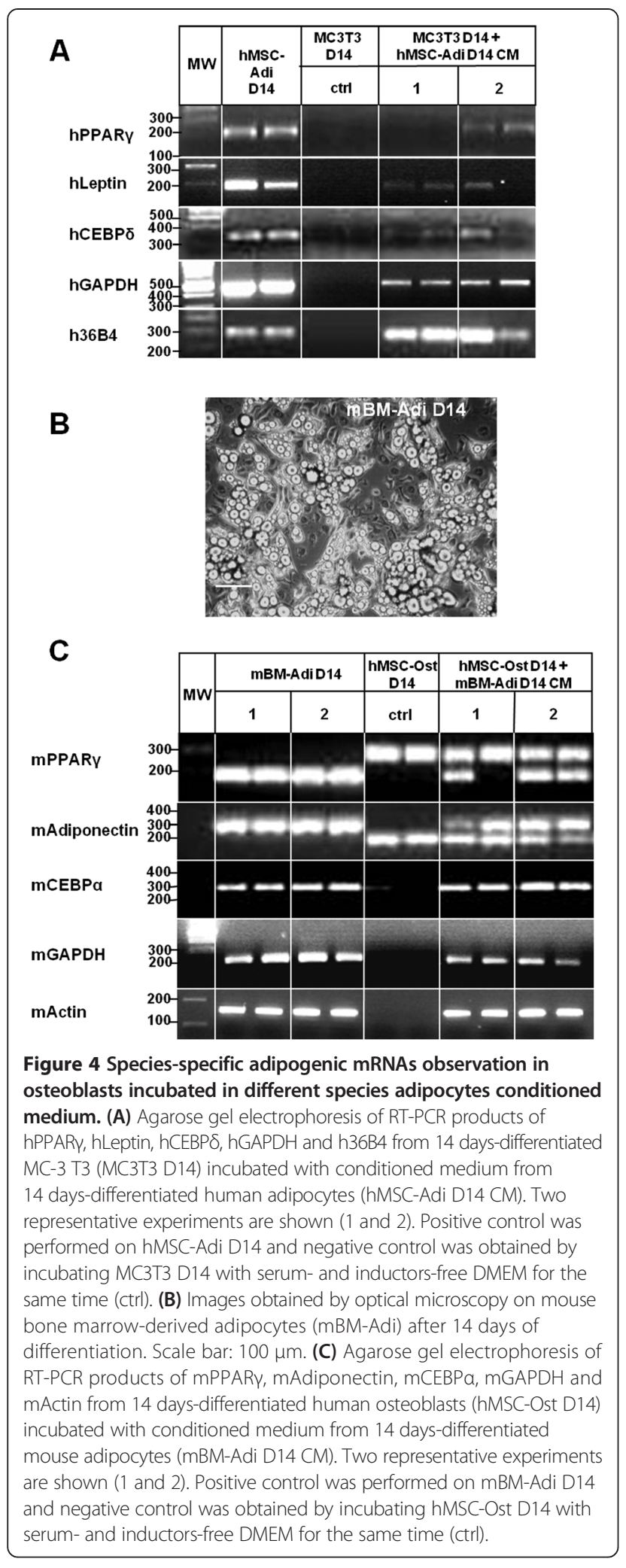

homogeneous in size, around $200 \mathrm{~nm}$, but their protein profile remains to be characterized to classify them either as exosomes or as ectosomes [36,37]. Both are membrane bound vesicles that differ based on their process of biogenesis, and biophysical properties, such as size and surface protein markers.

A significant increase of PPAR $\gamma, \mathrm{CEBP} \alpha$ and CEBP $\delta$ expression in osteoblasts incubated with hAdi-CM could be measured, suggesting an adipocyte-specific mRNA transfer. This hypothesis is corroborated by: (i) the presence of these transcripts in EVs, (ii) the fact that silencing of PPAR $\gamma$ mRNA in donor adipocytes inhibits the increase of PPAR $\gamma$ mRNA observed in the recipient osteoblasts after incubation in hAdi CM and (iii) the interspecies experiments, except for hCEBP $\alpha$ to mouse cells and mCEBP $\delta$ to human cells because of difficulties to optimize specific PCR. Besides, the genes used as controls, GAPDH, 36B4 and Actin, that are known to be found in EVs [19], are also transferred to their target cells (Figure 4A and C), corroborating the hypothesis of transcripts passage through EVs. Nonetheless, the bands observed in MC3T3 D14 incubated with hAdi D14 CM are slight and poorly reproducible, especially for hPPAR $\gamma$ and hLeptin. This can be explained by the fact that only a small quantity of human transcripts is transferred. For this reason, the observed amplification should be considered as qualitative more than quantitative. Concerning adiponectin, a time-dependent induction of its expression in hAdi could be measured, but no increase could be observed in human osteoblasts incubated with hAdi-CM (data not shown). However, a transfer of mouse adiponectin mRNA could be detected in hOst incubated with CM mBM-Adi by specific PCR.

An increase of miR-30c and miR-31 miRNAs, targeting osteogenic transcripts such as RUNX2 and Osterix, [23,26-28] and of miR-125a known to be significantly downregulated during osteogenic differentiation in human adipose-derived stem cells [38] and predicted to target the osteogenic genes Smad 2 and 4 [39], could be found in hOst incubated with hAdi-CM. However, no decrease of the expression of Runx 2 and Osterix could be observed (data not shown) as described in studies using cells overexpressing these miRNAs [23,25,27,28]. In our case, the slight induction of each miRNA observed in hOst incubated with hAdi-CM is maybe not sufficient to reproduce a significant repression of Runx2 and Osterix. Nonetheless we could detect a small decrease of the late osteoblastic marker transcripts $\mathrm{OC}$ et $\mathrm{OP}$. These were found to be targeted by miR-125b and miR-138 [23,40], two miRNAs that we have shown to be increased in hOst incubated with hAdi-CM, suggesting that the transferred miRNAs are functional. However their putative role in our model should be investigated in depth.

As RNA transfer between hMSC-derived adipocytes and hMSC-derived osteoblasts could be shown, we further examined whether EVs were directly implicated. 
hMSC-Ost were incubated with EVs isolated from hAdi$\mathrm{CM}$ and no adipogenic RNA increase could be found in hMSC-Ost (data not shown). This result might be attributed to the loss of part of the EVs and/or of their functional effect during the isolation procedure. Another possible explanation is that EVs are not the only way in which adipocytes may influence osteoblast gene expression and maybe some adipocytes-secreted molecules are needed in the medium to enhance their effects. Further study is required to answer these questions.

\section{Conclusions}

Here, we have shown for the first time that RNA transfer is possible between hMSC-derived adipocytes and osteoblasts through EVs. Additional studies are needed to clarify if this mechanism has a role in the adipocytic switch driven on osteoblasts by adipocytes inside bone marrow and if EVs could be a target component to regulate the competition between osteoblasts and adipocytes in the prevention or in the therapy of osteoporosis and other osteopenia.

\section{Methods \\ Cell culture experiments \\ Human MSC culture and induction of osteogenic and adipogenic differentiation}

Purified MSCs were purchased from Lonza (Verviers, Belgium) and were cultured as described previously [18]. Differentiation experiments were started when hMSCs had reached confluence (D0). To induce osteogenesis, hMSCs were cultured in DMEM with $10 \%$ FCS supplemented with osteogenic inductors $(50 \mu \mathrm{M}$ ascorbic acid, $10 \mathrm{mM} \beta$-glycerophosphate and $10^{-8} \mathrm{M}$ vitamin D3 (Sigma-Aldrich Corporation, St Quentin Fallavier, France)) for 14 days (hMSC-Ost). For adipogenic differentiation, hMSCs were cultured in DMEM with 10\% FCS supplemented with adipogenic inductors $(0.5 \mu \mathrm{M}$ dexamethasone, $0.5 \mathrm{mM}$ isobutyl-1-methylxanthine and $50 \mu \mathrm{M}$ indomethacin (Sigma-Aldrich Corporation)) for 0, 7, 14 or 21 days (hMSC-Adi).

\section{MC3T3 culture and osteogenic differentiation}

The MC3T3-E1 cells were plated in expansion medium composed of alpha-Modified Eagle Medium ( $\alpha$-MEM) (Dutscher) containing 10\% FCS, 1\% penicillin/streptomycin and $1 \%$ glutamine (Dutscher). Osteogenesis differentiation was started when MC3T3 had reached confluence (day 0) by incubation with osteogenic inductors $(50 \mu \mathrm{g} / \mathrm{ml}$ ascorbic acid and $10 \mathrm{mM} \beta$-glycerophosphate (Sigma-Aldrich Corporation) for 14 days.

\section{Primary mice bone marrow cell culture and adipogenic differentiation}

Female C57BL6 mice, 7 weeks of age, were purchased from Charles River and were acclimatized for 1 week under standard laboratory conditions. Immediately after sacrifice, femurs and tibias were removed and cleaned of connective tissue, the ends were cut, and the marrow was flushed with $\alpha$-MEM (PAN Biotech, Dutsher) supplemented with 15\% FCS (PAN Biotech), $2 \mathrm{mM}$ of glutamine (PAN Biotech), $50 \mathrm{IU} / \mathrm{ml}$ of penicillin (PAN Biotech), and $50 \mu \mathrm{g} / \mathrm{ml}$ of streptomycin (PAN Biotech). Single-cell suspensions were prepared in $\alpha-M E M$ by drawing the cells several times through graded needles. Cell density was determined using a Malassez counting chamber. For all experiments, cells were plated at a density of $2 \times 10^{6}$ cells $/ \mathrm{cm}^{2}$ and incubated at $37^{\circ} \mathrm{C}$ in $5 \%$ $\mathrm{CO}_{2}$. After 24 hours, non-adherent cells were removed and the medium was changed every 3 days until cell confluence.

Adipogenesis was induced by DMEM (PAN Biotech) $10 \%$ FCS supplemented with $10 \mu \mathrm{g} / \mathrm{ml}$ insulin/0,5 $\mu \mathrm{M}$ dexamethasone/100 $\mu \mathrm{M}$ indomethacin/500 $\mu \mathrm{M}$ 3-isobutyl1-methylxanthine (Sigma-Aldrich Corporation) for 4 days and maintained in $10 \mu \mathrm{g} / \mathrm{ml}$ insulin $/ 0,5 \mu \mathrm{M}$ dexamethasone/5 $\mu \mathrm{M}$ pioglitazone (Sigma-Aldrich Corporation) for 10 days (mBM-Adi).

\section{Conditioned medium (CM) experiments}

After adipogenic differentiation of hMSCs or mouse bone marrow derived cells for the indicated times, differentiation medium was discarded and replaced with serum- and inductors-free DMEM containing antibiotics for $48 \mathrm{~h}$ and collected (hAdi-CM or mBM-Adi CM). $1 \mathrm{ml}$ of CM was then applied on DMEM rinsed hMSCor MC3T3-derived osteoblasts cultured in 12-well plates and incubated for $48 \mathrm{~h}$. As controls, osteoblasts were incubated with serum- and inductors-free DMEM for the same time.

\section{Small interfering RNA (siRNA) transfection}

RNA interference was used to knockdown the expression of PPAR $\gamma$ in hMSC-Adi. siRNA for PPAR $($ siPPAR $\gamma$ ) and non-target controls were designed and synthesized by Dharmacon (ABgene Ltd, United Kingdom) and distributed by Thermo Fisher Scientific (PPARG Accell SMART pool, E-003436 and Accell control siRNA kit, K005000). hAdi D14 were transfected with siRNAs $(1 \mu \mathrm{M})$ for $72 \mathrm{~h}$ according to the manufacturer's instructions.

hMSC-Ost were incubated $48 \mathrm{~h}$ in conditioned medium from hMSC-Adi transfected (hAdi D14 siPPAR $\gamma$-CM) or not (hAdi-CM) with siPPAR $\gamma$.

\section{Extracellular vesicles (EVs) isolation}

hMSC-derived adipocytes (hMSC-Adi) at the indicated times of differentiation (D0, D7, D14, D21) were incubated in EVs-free DMEM and the obtained conditioned medium from $1.5 \times 10^{7}$ cells was collected after $48 \mathrm{~h}$. EVs were purified by differential centrifugation and 
concentration as previously described [29]. Briefly, hMSCAdi-conditioned medium (hAdi-CM) was centrifuged for $10 \mathrm{~min}$ at $2000 \mathrm{~g}$ to eliminate cell contamination. Supernatants were further centrifuged for $20 \mathrm{~min}$ at $3500 \mathrm{~g}$, filtered through a $0.45 \mu \mathrm{m}$ filter and concentrated thanks to Amicon Ultra-15 Centrifugal Filter Units with $10 \mathrm{kDa}$ cut-off (Millipore). EVs were pelleted by ultracentrifugation at 110,000 g for $70 \mathrm{~min}$. The EV pellets were either resuspended in DMEM, or lysed with Extract-all for RNA

Table 1 mRNA primer sequences

\begin{tabular}{|c|c|c|}
\hline mRNA & Primers & $\begin{array}{l}\text { Accession } \\
\text { numbers }\end{array}$ \\
\hline \multicolumn{3}{|l|}{$\begin{array}{l}\text { Human specific } \\
\text { primers }\end{array}$} \\
\hline \multirow[t]{2}{*}{ hPPARY } & F: 5'-GCTTCTGGATTTCACTATGG-3' & NM_005037 \\
\hline & R: 5'-AAACCTGATGGCATTATGAG-3' & \\
\hline \multirow[t]{2}{*}{ hLeptin } & F: 5'-ATTTCACACACGCAGTCAGT-3' & NM_00230 \\
\hline & R: 5'-GAAGAAGATCCCGGAGGT-3' & \\
\hline \multirow[t]{2}{*}{ hCEBPa } & F: 5'-ACTGGGACCCTCAGCCTTG-3' & NM_004364 \\
\hline & R: 5'-TGGACTGATCGTGCTTCGTG-3' & \\
\hline \multirow[t]{2}{*}{ hCEBPS } & F: 5'-ACGACGACGAGAGCGCCATC-3' & NM_005195 \\
\hline & R: 5'-CGCCCGCCTTGTGATTGC-3' & \\
\hline \multirow[t]{2}{*}{ hOC } & F: 5'-ATGAGAGCCCTCACACTCCTC-3' & NM_199173 \\
\hline & R: 5'-GCCGTAGAAGCGCCGATAGGC-3' & \\
\hline \multirow[t]{2}{*}{$h O P$} & F: 5'-CCTGCCAGCAACCGAAGTIT-3' & NM_001040058.1 \\
\hline & R: 5'-ACTGTCCTTCCCACGGCTGT-3' & \\
\hline \multirow[t]{2}{*}{ hYWHAZ } & F: 5'-GGTCATCTTGGAGGGTCGTC-3' & NM_145690 \\
\hline & R: 5'-GTCATCACCAGCGGCAAC-3' & \\
\hline \multirow[t]{2}{*}{ hGAPDH } & F: 5'-GTTCCAATATGATTCCACCC-3' & NM_002046.5 \\
\hline & R: 5'-AGGGATGATGTTCTGGAGAG-3' & \\
\hline \multirow[t]{2}{*}{ h36B4 } & F: 5'-CGACCTGGAAGTCCAACTAC-3' & NM_001002.3 \\
\hline & R: 5'-AGCAACATGTCCCTGATCTC-3' & \\
\hline \multicolumn{3}{|l|}{$\begin{array}{l}\text { Mouse specific } \\
\text { primers }\end{array}$} \\
\hline \multirow[t]{2}{*}{ mPPARY } & F: 5'-TITTCAAGGGTGCCAGTTTC-3' & NM_001127330 \\
\hline & R: 5'-AATCCTTGGCCCTCTGAGAT-3' & \\
\hline \multirow[t]{2}{*}{ mAdiponectin } & F: 5'-CCCAGTCATGCCGAAGA-3' & NM_009605.4 \\
\hline & R: 5'-TACATTGGGAACAGTGACGC-3' & \\
\hline \multirow[t]{2}{*}{$m C E B P a$} & F: 5'-TTACAACAGGCCAGGTTTCC-3' & NM_007678 \\
\hline & R: 5'-CTCTGGGATGGATCGATTGT-3' & \\
\hline \multirow[t]{2}{*}{ mGAPDH } & F: 5'-GGCATTGCTCTCAATGACAA-3' & NM_008084 \\
\hline & R: 5'-TGTGAGGGAGATGCTCAGTG-3' & \\
\hline \multirow[t]{2}{*}{ mActin } & F: 5'-AATTTCTGAATGGCCCAGGT-3' & NM_007393 \\
\hline & R: 5'-GGTAAGGTGTGCACTTTTATTGG-3' & \\
\hline
\end{tabular}

Shown are the primer sequences and Genbank accession numbers. F: forward; R: reverse. $h$ : human; $m$ : mouse. PPARy, peroxisome proliferator-activated receptor gamma; CEBPa and $\delta, C C A A T / e n h a n c e r$ binding protein alpha and delta; YWHAZ, tyrosine 3-monooxygenase/tryptophan 5- monooxygenase activation protein, GAPDH, Glyceraldehyde 3-phosphate dehydrogenase. Human and mouse primers for PPAR $y$ amplified isoforms 1 and 2. extraction or washed with PBS, pelleted again and resuspended in PBS.

\section{RNA expression measurement RNA isolation}

Total RNA was extracted using Extract-All reagent (Eurobio, Les Ulis, France), according to the manufacturer's instructions. Total RNA was digested with DNase I (Roche Diagnostics, Meylan, France) and quantified by Nanodrop at $260 \mathrm{~nm}$ wavelength.

\section{mRNAs expression analysis}

Total RNA was reverse transcribed using Maxima FirstStrand cDNA Synthesis kit (Fermentas, Thermoscientific, France), and subjected to quantitative real-time PCR on the LightCycler ${ }^{\circ}$ Carousel-Based System (Roche Diagnostics) using the LightCycler ${ }^{\circ}$ Fast Start DNA Master SYBR ${ }^{\circ}$ Green I kit as described previously and specific primers designed using Oligo 6 software (MedProbe, Oslo, Norway) (Table 1) [16]. Results were normalized against expression of the indicated genes and compared to the expression level as baseline by the 2- $\Delta \Delta \mathrm{Ct}$ method, and log2-transformed fold changes of normalized 2-deltaCT.

For interspecies experiments, subsequent PCR products were migrated on $1.8 \%$ agarose gel for visualization.

\section{miRNA expression analysis}

Total RNA was reverse transcribed using the miScript II RT Kit (Qiagen), and subjected to quantitative real-time PCR on the LightCycler ${ }^{\circ}$ Nano System (Roche Diagnotics) using the miScript SYBR Green PCR Kit (Qiagen) according to the manufacturer's instructions. Specific primers are listed in Table 2. Results were normalized against RNU6P expression and compared to the expression level as baseline by the 2- $\Delta \Delta \mathrm{Ct}$ method, and log2-transformed fold changes of normalized 2-deltaCT.

\section{Electron microscopy of EVs}

EVs from hMSC-Adi were labeled as described by Thery et al. [29]. Briefly, they were fixed in $2 \%$ paraformaldehyde in PBS, loaded onto formvar carbon-coated grids and incubated during 20 minutes. After extensive

Table 2 miRNA primer sequences

\begin{tabular}{lll}
\hline miRNA & Primers & $\begin{array}{l}\text { Reference or } \\
\text { accession number }\end{array}$ \\
\hline miR-30c & 5'-TGTAAACATCCTACACTCTCAGC-3' & {$[25]$} \\
miR-138 & 5'-GCTATTTCACGACACCAGGGTT-3' & NR_029680.1 \\
miR-125a & 5'-GGTCCCTGAGACCCTTAACCT-3' & NR_029694.1 \\
miR-125b & 5'-TCCCTGAGACCCTAACTTGTGA-3' & NR_029693.1 \\
miR-31 & 5'-GGAGGCAAGATGCTGGCATA-3' & NR_029505.1 \\
RNU6P & 5'-GTGCTCGCTTCGGCAGCACATAT-3' & NR_004394.1 \\
\hline
\end{tabular}

Shown are the primer sequences and Genbank accession numbers. 
washing with PBS, EVs were postfixed in 1\% glutaraldehyde for $5 \mathrm{~min}$, extensively washed in distilled water and embedded in a mixture of uranyl acetate and methyl cellulose for negative coloration. They were then examined in a Zeiss 902, $80 \mathrm{kV}$ transmission electron microscope.

\section{Statistical analysis}

Results are expressed as median of experimental data, using quartiles [Q1;Q3] to evaluate variability around this value. All statistical comparisons were made using a nonparametric Mann-Whitney U-Test, using SPSS software version 18. Differences were considered significant at $\mathrm{p}<0.05\left(* \mathrm{p}<0.05,{ }^{* * *} \mathrm{p}<0.01\right)$.

\section{Abbreviations}

Adi: Adipocyte; BM: Bone Marrow; CEBPa and $\delta:$ CCAAT/enhancer binding protein alpha and delta; CM: Conditioned Medium; EVs: Extracellular Vesicles; GAPDH: Glyceraldehyde 3-phosphate dehydrogenase; hMSC: Human Mesenchymal Stem Cell; miRNAs: microRNAs; OC: Osteocalcin; Ost: Osteoblast; OP: Osteopontin; PPARY: Peroxisome proliferator-activated receptor gamma; RNAs: RiboNucleic Acids; RT-qPCR: Reverse Transcription-quantitative Polymerase Chain Reaction; YWHAZ: Tyrosine 3-monooxygenase/tryptophan 5- monooxygenase activation protein.

\section{Competing interests}

The authors declare that they have no competing interests.

\section{Authors' contributions}

Study design: PJM. Study conduct: PJM and OB. Data collection: PJM, NH and AC. Data analysis: PJM. Data interpretation: PJM. Primary mice bone marrow cell culture and adipogenic differentiation: OG and CC. Drafting manuscript: $\mathrm{PJM}, \mathrm{OB}$ and $\mathrm{OG}$. Revising manuscript: $\mathrm{OB}$ and $\mathrm{PH}$. Approving final version of manuscript: All authors. PJM and NH take responsibility for the integrity of the data analysis.

\section{Acknowledgements}

The authors thank Cécile ALLET and Anne LOYENS of the BICeL-Campus Lille 2 IMPRT Facility for access to instruments and technical advices. They also thank Dr. John Halket for the reading and English language corrections of the manuscript.

This work was supported by ULCO.

\section{Author details}

'Univ Lille Nord de France, F-59000 Lille, France. ²PMOI EA 4490, IFR 114, F-62327 Boulogne sur Mer and F-59000, Lille, France. ${ }^{3}$ Université du Littoral Côte d'Opale, ULCO, F-62327 Boulogne sur Mer, France. ${ }^{4}$ UCEIV EA 4492, F-59140 Dunkerque, France. ${ }^{5}$ Université du Littoral Côte d'Opale, EA4492 Unité de Chimie Environnementale et Interactions sur le Vivant (UCEIV) Maison de la Recherche en Environnement Industriel 2, ULCO, 189A, Avenue Maurice Schumann, 59140 Dunkerque, France.

Received: 9 December 2014 Accepted: 24 February 2015

Published online: 18 March 2015

\section{References}

1. Duque G. Bone and fat connection in aging bone. Curr Opin Rheumatol. 2008:20:429-34.

2. Gimble JM, Zvonic S, Floyd ZE, Kassem M, Nuttall ME. Playing with bone and fat. J Cell Biochem. 2006;98:251-66.

3. Justesen J, Stenderup K, Ebbesen EN, Mosekilde L, Steiniche T, Kassem M. Adipocyte tissue volume in bone marrow is increased with aging and in patients with osteoporosis. Biogerontology. 2001;2:165-71.

4. Martin TJ. Bone biology and anabolic therapies for bone: current status and future prospects. J Bone Metab. 2014:21:8.

5. Meunier P, Aaron J, Edouard C, Vignon G. Osteoporosis and the replacement of cell populations of the marrow by adipose tissue. A quantitative study of 84 iliac bone biopsies. Clin Orthop. 1971;80:147-54.
6. Zhao J-W, Gao Z-L, Mei H, Li Y-L, Wang Y. Differentiation of human mesenchymal stem cells: the potential mechanism for estrogen-induced preferential osteoblast versus adipocyte differentiation. Am J Med Sci. 2011;341:460-8.

7. Ecklund K, Vajapeyam S, Feldman HA, Buzney CD, Mulkern RV, Kleinman PK, et al. Bone marrow changes in adolescent girls with anorexia nervosa. J Bone Miner Res Off J Am Soc Bone Miner Res. 2010;25:298-304.

8. Georgiou KR, Hui SK, Xian CJ. Regulatory pathways associated with bone loss and bone marrow adiposity caused by aging, chemotherapy, glucocorticoid therapy and radiotherapy. Am J Stem Cells. 2012;1:205-24.

9. Zayzafoon M, Gathings WE, McDonald JM. Modeled microgravity inhibits osteogenic differentiation of human mesenchymal stem cells and increases adipogenesis. Endocrinology. 2004;145:2421-32.

10. Hu R, Liu W, Li H, Yang L, Chen C, Xia Z-Y, et al. A Runx2/miR-3960/miR-2861 regulatory feedback loop during mouse osteoblast differentiation. J Biol Chem. 2011;286:12328-39.

11. Li H, Xie H, Liu W, Hu R, Huang B, Tan Y-F, et al. A novel microRNA targeting HDAC5 regulates osteoblast differentiation in mice and contributes to primary osteoporosis in humans. J Clin Invest. 2009;119:3666-77.

12. Kawai M, de Paula FJA, Rosen CJ. New insights into osteoporosis: the bone-fat connection. J Intern Med. 2012;272:317-29.

13. Sadie-Van Gijsen H, Crowther NJ, Hough FS, Ferris WF. The interrelationship between bone and fat: from cellular see-saw to endocrine reciprocity. Cell Mol Life Sci CMLS. 2013;70:2331-49.

14. Scheideler M, Elabd C, Zaragosi L-E, Chiellini C, Hackl H, Sanchez-Cabo F, et al. Comparative transcriptomics of human multipotent stem cells during adipogenesis and osteoblastogenesis. BMC Genomics. 2008;9:340.

15. Kollmer M, Buhrman JS, Zhang Y, Gemeinhart RA. Markers are shared between adipogenic and osteogenic differentiated mesenchymal stem cells. J Dev Biol Tissue Eng. 2013;5:18-25.

16. Ponce ML, Koelling S, Kluever A, Heinemann DEH, Miosge N, Wulf G, et al. Coexpression of osteogenic and adipogenic differentiation markers in selected subpopulations of primary human mesenchymal progenitor cells. J Cell Biochem. 2008;104:1342-55.

17. Tontonoz P, Spiegelman BM. Fat and beyond: the diverse biology of PPARY. Annu Rev Biochem. 2008;77:289-312.

18. Clabaut A, Delplace S, Chauveau C, Hardouin P, Broux O. Human osteoblasts derived from mesenchymal stem cells express adipogenic markers upon coculture with bone marrow adipocytes. Differentiation. 2010;80:40-5.

19. Valadi H, Ekström K, Bossios A, Sjöstrand M, Lee JJ, Lötvall JO. Exosomemediated transfer of mRNAs and microRNAs is a novel mechanism of genetic exchange between cells. Nat Cell Biol. 2007;9:654-9.

20. Aoki N, Jin-no S, Nakagawa Y, Asai N, Arakawa E, Tamura N, et al. Identification and characterization of microvesicles secreted by 3 T3-L1 adipocytes: redoxand hormone-dependent induction of milk fat globule-epidermal growth factor 8-associated microvesicles. Endocrinology. 2007;148:3850-62.

21. Müller G, Schneider M, Biemer-Daub G, Wied S. Microvesicles released from rat adipocytes and harboring glycosylphosphatidylinositol-anchored proteins transfer RNA stimulating lipid synthesis. Cell Signal. 2011;23:1207-23.

22. Ogawa R, Tanaka C, Sato M, Nagasaki H, Sugimura K, Okumura K, et al. Adipocyte-derived microvesicles contain RNA that is transported into macrophages and might be secreted into blood circulation. Biochem Biophys Res Commun. 2010;398:723-9.

23. Eskildsen T, Taipaleenmäki H, Stenvang J, Abdallah BM, Ditzel N, Nossent AY, et al. MicroRNA-138 regulates osteogenic differentiation of human stromal (mesenchymal) stem cells in vivo. Proc Natl Acad Sci U S A. 2011;108:6139-44.

24. Wu T, Zhou H, Hong $Y$, Li J, Jiang $X$, Huang $H$. miR-30 family members negatively regulate osteoblast differentiation. J Biol Chem. 2012;287:7503-11.

25. Zhang Y, Xie R-L, Gordon J, LeBlanc K, Stein JL, Lian JB, et al. Control of mesenchymal lineage progression by microRNAs targeting skeletal gene regulators Trps1 and Run×2. J Biol Chem. 2012;287:21926-35.

26. Zhang Y, Xie R-L, Croce CM, Stein JL, Lian JB, van Wijnen AJ, et al. A program of microRNAs controls osteogenic lineage progression by targeting transcription factor Runx2. Proc Natl Acad Sci U S A. 2011;108:9863-8.

27. Baglio SR, Devescovi V, Granchi D, Baldini N. MicroRNA expression profiling of human bone marrow mesenchymal stem cells during osteogenic differentiation reveals Osterix regulation by miR-31. Gene. 2013;527:321-31.

28. Goettsch C, Rauner M, Pacyna N, Hempel U, Bornstein SR, Hofbauer LC. miR-125b regulates calcification of vascular smooth muscle cells. Am J Pathol. 2011;179:1594-600. 
29. Ragni E, Viganò M, Rebulla P, Giordano R, Lazzari L. What is beyond a qRT-PCR study on mesenchymal stem cell differentiation properties: how to choose the most reliable housekeeping genes. J Cell Mol Med. 2013;17:168-80

30. Théry C, Amigorena S, Raposo G, Clayton A. Isolation and characterization of exosomes from cell culture supernatants and biological fluids. Curr Protoc Cell Biol Editor Board Juan Bonifacino Al. 2006;Chapter 3:Unit 3.22.

31. Muruganandan S, Roman AA, Sinal CJ. Adipocyte differentiation of bone marrow-derived mesenchymal stem cells: Cross talk with the osteoblastogenic program. Cell Mol Life Sci. 2009;66:236-53.

32. Elbaz A, Wu X, Rivas D, Gimble JM, Duque G. Inhibition of fatty acid biosynthesis prevents adipocyte lipotoxicity on human osteoblasts in vitro. J Cell Mol Med. 2010;14:982-91.

33. Maurin A, Chavassieux P, Frappart L, Delmas P, Serre C, Meunier P. Influence of mature adipocytes on osteoblast proliferation in human primary cocultures. Bone. 2000;26:485-9.

34. Maurin AC, Chavassieux PM, Vericel E, Meunier PJ. Role of polyunsaturated fatty acids in the inhibitory effect of human adipocytes on osteoblastic proliferation. Bone. 2002;31:260-6.

35. Camussi G, Deregibus MC, Bruno S, Cantaluppi V, Biancone L. Exosomes/ microvesicles as a mechanism of cell-to-cell communication. Kidney Int. 2010;78:838-48.

36. Choi D-S, Kim D-K, Kim Y-K, Gho YS: Proteomics of extracellular vesicles: Exosomes and ectosomes. Mass Spectrom Rev 2014.

37. Lee Y, Andaloussi SE, Wood MJA. Exosomes and microvesicles: extracellular vesicles for genetic information transfer and gene therapy. Hum Mol Genet. 2012;21:R125-34

38. Zhang Z, Zhang $H$, Kang $Y$, Sheng $P$, Ma Y, Yang Z, et al. miRNA expression profile during osteogenic differentiation of human adipose-derived stem cells. J Cell Biochem. 2012;113:888-98.

39. Gong Y, Xu F, Zhang L, Qian Y, Chen J, Huang H, et al. MicroRNA expression signature for Satb2-induced osteogenic differentiation in bone marrow stromal cells. Mol Cell Biochem. 2014;387:227-39.

40. Huang K, Fu J, Zhou W, Li W, Dong S, Yu S, et al. MicroRNA-125b regulates osteogenic differentiation of mesenchymal stem cells by targeting Cbf $\beta$ in vitro. Biochimie. 2014;102:47-55.

\section{Submit your next manuscript to BioMed Central and take full advantage of:}

- Convenient online submission

- Thorough peer review

- No space constraints or color figure charges

- Immediate publication on acceptance

- Inclusion in PubMed, CAS, Scopus and Google Scholar

- Research which is freely available for redistribution 